\title{
Investigation and Analysis of Anxiety Dissemination in Various Situations and Research on Methods of Eliminating Anxiety in Population
}

\section{Qiang Cao}

Macau University of Science and Technology School of Business Administration

Qi Zhang

Taishan University

Yi Qiang (D 462766753@qq.com)

Kunming University of Science and Technology

\section{Research Article}

Keywords: anxious emotions, emotional communication, questionnaire survey, micro-expressions

Posted Date: April 26th, 2021

DOl: https://doi.org/10.21203/rs.3.rs-461945/v1

License: (c) (1) This work is licensed under a Creative Commons Attribution 4.0 International License.

Read Full License 
Investigation and Analysis of Anxiety Dissemination in Various Situations and Research on

Methods of Eliminating Anxiety in Population

Qiang Cao ${ }^{1, \#}$, Qi Zhang ${ }^{2, \#}$, Yi Qiang ${ }^{3, *}$

${ }^{1}$ Macau University of Science and Technology School of Business Administration, Taipa, 999078, Macau, China.

${ }^{2}$ Taishan University, Human Resource Management, School of Business Administration, Tai'an, 271000, Shandong, China.

${ }^{3}$ Kunming University of Science and Technology, Kunming, 650000, Yunnan, China.

\#Qiang Cao and Qi Zhang are co-first authors

"Corresponding author: Yi Qiang, Kunming University of Science and Technology, Panlong District Huancheng East Road No. 50, Kunming, 650000, Yunnan, China.

\section{Email: 462766753@qq.com}

Tel: $+\mathbf{8 6}-13518716123$

\section{Abstract}

It is very common that people are affected by different levels of anxiety in different environments. However, there is no effective research or corresponding measures for this phenomenon. This study through the questionnaire survey combined with interviews and experiments, through the investigation and experiment to find out the reasons for the spread of anxiety in the group, and through further experiments found that people can produce positive emotions and eliminate anxiety when watching portraits with positive emotions, and finally find 
out the method to solve the problem of anxiety in private and public occasions.

Keywords: anxious emotions, emotional communication , questionnaire survey , micro-expressions

\section{INTRODUCTION}

People ' s psychological state is different in different environments [ 1 ], under the influence of some conditions, people will be more vulnerable to the environment of diffuse anxiety [ 2 ], or more easily spread their own anxiety. [ 3 ] At the same time, it will affect people ' $\mathrm{s}$ emotions and ultimately determine whether people will produce anxiety in some specific environments. The reasons include people 's educational background, age, working status [ 4 ], and the closeness or alienation of the relationship between people who spread anxiety and themselves in the environment [ 5 ]. These factors determine whether people will be affected by the anxiety spread in the environment and whether they will spread anxiety to the outside world. [ 6 ] If the duration of anxiety is too long, it may be transformed into symptoms such as emotional autonomic nerve dysfunction and emotional disorder. If the intervention of professionals cannot be obtained in time, it may eventually develop into suicide tendency.[ 7 ] [ 8 ] The atmosphere of anxiety in the environment is often derived from the anxiety of a certain body in the crowd itself for various reasons, when it produces anxiety, because the individual in the anxiety state of the micro expression and action will be captured by the surrounding people, because people have empathy, in this case the surrounding people will produce empathy effect, so that the surrounding people are affected by anxiety. Individuals affected by it are likely to produce anxiety and become another spread of anxiety. When there is a certain proportion between the individuals who transmit anxiety signals in the population and those who are affected by anxiety and have anxiety, it can be 
considered that there is anxiety in this group. This study investigated people's probability of being affected by anxiety in various places, and was committed to understanding the reasons for the different probability of being affected by anxiety in different places through interviews with participants. Through the combination of experiments and surveys, we found a method to prevent the spread of anxiety in the population, and finally proposed a scheme to prevent the spread of anxiety in private and public occasions.

\section{Background}

At present, group riots often occur in countries around the world. However, when government departments investigate the causes of these riots, they find that there are no clear reasons for some riots. Some riots occur only because someone in the group has anxiety for various reasons, which spreads throughout the population through certain channels, [ 9 ] resulting in most people infected by anxiety, some people have conflict with others under the influence of anxiety, and others will expand the conflict under the influence of anxiety, which eventually leads to the occurrence of group riots. Therefore, it is very important to study the causes of anxiety spread in the crowd and how to curb the spread of anxiety. This study can provide methods and suggestions for government departments and various institutions to curb the spread of anxiety in the crowd, [ 10 ] reduce the frequency of anxiety spread in the crowd, and then realize the purpose of maintaining community safety and reducing the frequency of riots.

\section{Survey results}


Through this survey and related interviews, we found that people with higher degree ( master and above ) are more likely to be affected by anxiety in public places or schools than those with lower education, and people with lower education are more likely to be affected by anxiety at home. Through interviews with different subjects, we found that subjects with lower education are more likely to be affected by anxiety transmitted by family members at home, and subjects with higher education are more likely to be affected by anxiety transmitted by working partners or classmates. Through SPSS analysis, it can be found that there is a significant correlation between the two ( Pearson correlation $=0.842$ and $p<0.05$ ), and most respondents believe that the probability of anxiety infection in the family and the spread of anxiety is higher than that in the public. More than half of the respondents said that they are susceptible to anxiety infection in the family. When the respondents are students in school, they are more likely to be affected by anxiety in parks, supermarkets, restaurants and other places. However, respondents who have jobs and who do not participate in work and are not students are less affected by anxiety in parks, supermarkets, restaurants and other occasions. After further interviews with relevant respondents, we know that some of the respondents who are students spend most of their time on campus and family, so when they come to parks, supermarkets, restaurants and other occasions, they are prone to tension, and people are more likely to be affected by anxiety in the crowd.However, respondents who have participated in the work and those who are temporarily unemployed often come to these occasions, so they are often in a relaxed state in these occasions. Therefore, compared with students, they are more vulnerable to anxiety in parks, supermarkets, restaurants and other occasions or spread anxiety. 


\section{movements}

Through the interview and questionnaire survey, we know that ' anxiety in the crowd ' is often spread in the form of language or body movements. People often show in facial expressions or body movements when they are anxious. Due to the empathy effect of people, other people in the crowd have a certain probability to produce anxiety because of empathy effect with the anxious [ 11 ] when they observe the micro-expressions or micro-movements of the anxious, and this person will also produce some micro-expressions and micro-movements that make other people produce anxiety after anxiety, [ 12 ] which makes anxiety further spread in the crowd and eventually cause anxiety in the whole population. It is inevitable for people to observe other people ' $s$ micro-expressions and produce empathy effect in the crowd, [13 ] while micro-actions made by people under the subconscious of anxiety also have the possibility of spreading anxiety. According to relevant studies, [ 14 ] people tend to make defensive actions or offensive actions unconsciously under anxiety, such as fist-shaking or slightly forward-tilting. [ 15 ] When other people in the crowd observe these actions of the anxious people, they will be vigilant against the surrounding environment unconsciously. [ 16 ] In the vigilant state, people's psychological pressure increases [ 17 ] and they are more likely to fall into anxiety. [ 18] In addition to the interference of the micro-expression of the anxious people in the crowd, [ 19 ] people are extremely prone to fall into anxiety in this environment. [ 20 ]

\section{Anxiety spreads in the crowd through language}

Usually people communicate effectively with their acquaintances or relatives [ 21 ]. People care more about relatives or acquaintances than strangers. When people ' s relatives or acquaintances fall 
into anxiety, people tend to explore the causes of their relatives into anxiety, which gives the opportunity to spread anxiety through language. [22 ] When people in anxiety communicate with others, their tone and tone are often quite different from those in peace, resulting in a certain sense of strangeness and alienation for those who communicate with them. The sense of strangeness and alienation will cause obstacles to communication, [ 23 ] and the obstacles to communication will increase the anxiety of both sides. [ 24 ] The reason for people' s anxiety is often that they encounter some difficult problems in life. If the relationship between the person communicating with the anxious person and the anxious person is relatives or friends, the communicator will subconsciously help the anxious person to find a solution to the problem or think about what kind of help they can provide. [ 25 ] If the communicator cannot solve the problems encountered by the anxious person or provide effective help to the anxious person, the communicator will also feel frustration and loss. [ 26 ] The frustration and loss are a major reason for people' $\mathrm{s}$ anxiety. Therefore, even for good intentions, communicating with the anxious person is still likely to transform the communicator without anxiety into the anxious person, and ultimately make the anxiety further spread in the population. At the same time, we interviewed some respondents who thought they wouldn 't be infected by ' language anxiety ', who thought they wouldn 't be infected by language anxiety in the process of filling out the questionnaire.

Through further interviews and tests, we found that in this part of the people who think they will not be infected by anxiety, some are not really not infected by anxiety in language. In order to ensure the accuracy of this survey, we will exclude this part of the respondents in the statistical survey results, and ultimately leave the respondents who will not be infected by anxiety in language. Through observation and interviews, we find that this part of the respondents who are not affected by language anxiety age polarization, namely children under 11 years of age and the elderly, 
children due to experience and other restrictions, difficult to find or understand the language carried by the anxiety, can not be affected. The reasons why the elderly are not affected by language anxiety include cognitive degradation caused by Alzheimer 's disease and other reasons, which makes them unable to correctly understand the meaning of the content contained in the language or have more experience, and the anxiety carried in the language is difficult to affect it. For young or middle-aged people, because they are usually able to correctly understand and find anxiety in language and because of their experience or personality and other reasons, they cannot invalidate the anxiety carried in other people 's language, and ultimately make the language anxiety affect it, and ultimately produce anxiety itself, and even become the communicator of anxiety.

\section{Exploring the reasons why people are more likely to spread anxiety at home}

Through questionnaires and further interviews, the team found that anxiety was more likely to spread in people ' $\mathrm{s}$ families than outside. Through interviews, we know that people are affected by anxiety at home and the reasons for anxiety are often due to the quarrel of minor matters or the nagging of spouses. [ 27 ] In public situations, people often do not have anxiety in the face of the same things. According to relevant surveys, people are often relaxed when they are at home. Contrary to traditional cognition, [ 28 ] when people relax, if there are some trivial things that affect their mood, it will not be as ' careless ' in traditional cognition but will be more likely to feel anxious because of these things. In the face of what relatives do to make them uncomfortable, people will not have more patience with relatives as in traditional cognition, but are more likely to be irritated by what close people do not conform to their wishes. [ 29 ] Through interviews, we learn that a gentleman who has been careful at work has never been in conflict with his colleagues or boss in decades of work. In the eyes of his colleagues and boss, he is a man who has never been 
in conflict with or anxious. He has never been stressed in the face of work pressure, but the gentleman says he can 't tolerate his wife 's nagging about him or his children 's failure in grades. When he comes home, his wife and children 's small mistakes or unpleasant things can make he anxious.When communicating with his wife and children, the tone of this gentleman is more irritable than when communicating with colleagues or bosses at work. Interestingly, when his boss and colleagues go to the house to visit the gentleman, the gentleman can use a good attitude in the workplace to treat his wife and children, but when his colleagues and the boss leave, the gentleman will be anxious at home because of a variety of small things. For the reasons, the gentleman revealed to us that he was in a state of ' tension ' in public and ' relaxed ' when he had only family members at home. When he was in a working state, it was difficult for him to feel anxious about what happened around him. When he had only family members at home, he would feel more relaxed. But at the same time, some small things that made him uncomfortable would also make him very anxious, and do not hide these emotions, so that anxiety spread in his family. Among other respondents, the same situation as this gentleman is very common. People are often in a state of relaxation at home, and the threshold of people ' $\mathrm{s}$ anxiety in a state of relaxation is lower than that in a public situation. People often do not conceal their anxiety in a state of relaxation, and the anxiety without concealment is more likely to affect the mental status of others. Therefore, people are more likely to generate or disseminate anxiety at home.

\section{Influence of age on anxiety of respondents}

According to the survey of the degree of anxiety affected by the respondents at all ages, we found that most of the respondents in the ' when people around have anxiety and other negative emotions, 
whether the respondents will be affected by their anxiety, according to the severity of the impact score, if the impact is serious, take the highest 10 points, if there is no impact, take 0 points, if there is an impact, according to the degree of influence between 0 and 10 points ' in this problem, the score is concentrated in the range of 7-10 points. After further statistical work in the investigation group, we can find that the age of the respondents who scored 7-10 points in this problem is concentrated in the range of $18-40$ years old. In order to determine whether there is a correlation between the two, the investigation group uses SPSS software to examine its correlation, and the reliability and validity of the two groups of data are tested, and the correlation is strong ( Pearson = $0.753 \mathrm{p}<0.05)$. After further interviews, the team learned that the 11-40 age group had a high level of cognitive ability to correctly understand the anxiety contained in the language of others, and that this age group had greater learning or working pressure than other age groups. Therefore, the impact of anxiety transmitted in this age group was significantly higher than that of people under 18 or over 40.Interestingly, most of the respondents who think that the anxiety of the crowd has no effect on themselves ( this topic chooses 0 points ) are over 65 years old. Many of the respondents in this age group think that they have a deeper understanding of life and youth, which makes them not easily affected by the external environment. Among the respondents under 11 years old, many of them are limited by their own cognitive ability and understanding ability, and it is difficult to find other people ' s anxiety in language and action. Therefore, the respondents of this age group are less affected by the anxiety in the crowd. 
From the perspective of organizational behavior, the spread of anxiety in the population is due to people 's herd behavior [ 30 ] People treat the information observed in the population ( including the language and behavior of others ) as ' information ' [ 31 ], which contains the anxiety of its source, and ultimately affects the recipient in the process of information processing, while transforming the person receiving the anxiety information into another communicator. In order to solve the problem of anxiety spread in the population, Zhang Qi, a member of the study group, designed and carried out an experiment. During the experiment, 20 volunteers were asked to observe the anxiety or positive emotions such as joy and love expressed by dynamic human images on TV screen or projection screen. During the experiment, the subjects' faces were photographed by a fast camera to observe the micro-expression changes. The CASME II dataset was used to analyze the emotional changes contained in the micro-expressions. The experimental group found that anxiety, joy, love and other positive emotions such as the dynamic human image in the TV screen or projection screen can affect the emotions of the subjects, and there is no significant difference between the influence of this kind of emotion and the real person in front of the subjects. After further experiments, the subjects with anxiety were brought into the screen with sixteen screens at the same time and each screen played dynamic characters with emotions of joy, love and anxiety, irritability and disappointment respectively. The screen with positive emotions and the screen with negative emotions were placed on the left and right sides of the room.

When the subjects enter the room, they will not be disturbed by any external interference. After the subjects entered the room, 200FPS high-speed camera was used to shoot the front of the subjects in the room. In order to ensure that the shooting equipment does not affect the subjects, the camera placed in the hidden part of the ceiling will not be found by the subjects. The facial resolution in the video clip can reach about $290 * 340$ pixels. After the experiment, the CASME II dataset is used to 
analyze the micro-expression at each time in the experiment. After the experiment, we found that the subjects were more inclined to watch the screens with positive emotional characters. After watching the dynamic portrait with positive emotions, we found that the subjects ' emotions have improved significantly by observing the subjects ' micro-expressions. The emotions of static characters appearing in posters and billboards are also considered to have an impact on people ' $\mathrm{s}$ emotions, but the impact is slightly lower than that of dynamic pictures and other people ' $\mathrm{s}$ emotions in groups. Therefore, when decoration is carried out in the family, photographs such as the whole family happiness or happy moments can be suspended in the obvious position of the family, which can effectively reduce the probability of anxiety generation and dissemination at home. Putting more billboards or dynamic screens depicting characters with emotions as joy and love in public occasions can effectively reduce the incidence of group anxiety communication events. At the same time, combined with big data analysis technology, some contents with positive emotional guidance can be pushed for people who may have anxiety to alleviate their anxiety.[34], Eventually reduce the possibility of anxiety spread in the group.

\section{Conclusion}

Anxiety is often transmitted through language and action in the population. People are more susceptible to anxiety at home than the outside world. [ 32 ] The premise of infection of anxiety is to correctly understand the emotions existing in language and action and cannot invalidate the anxiety contained in information. Therefore, young people and middle-aged people are more likely to be affected by the anxiety transmitted in the population. There is empathy effect between people, empathy effect is the basis of the spread of anxiety in the crowd, but through the empathy effect can also prevent the spread of anxiety in the crowd or eliminate the anxiety of the individual who produces anxiety. After investigation, we found that compared with the individual who observes the 
existence of anxiety, the individual who observes the positive emotion has a higher priority, and the billboards, electronic screens containing positive emotions, or static or dynamic can effectively alleviate the individual ' $\mathrm{s}$ anxiety and interfere with the individual ' $\mathrm{s}$ observation of individuals with anxiety in the crowd, which can prevent the spread of anxiety. Advertisements containing images of characters with positive emotions are installed in the public, or dynamic images of characters with positive emotions are displayed on the electronic screen in the public, and even big data technology is used to push some content containing positive information for individuals who may have anxiety, which can effectively alleviate the anxiety of individuals and prevent the spread of anxiety in the population.

\section{$X$. Methods and process of investigation and experiment}

In this experiment, questionnaires and interviews were combined. Before the beginning of the questionnaire survey, Qiang Cao, a member of the research group, designed a questionnaire on whether the respondents will be affected by the anxiety in the crowd and whether they will spread anxiety in various occasions when anxiety occurs. In order to verify the accuracy of the questionnaire, the research group randomly selected 50 volunteers who were willing to participate in this study. At present, it is internationally recognized that the SAS scale can effectively test the degree of anxiety of people. Therefore, the SAS scale was compared with the questionnaire made by Qiang Cao, a member of the research group, to determine whether the questionnaire made by Qiang Cao was effective. SAS scale was used to measure the anxiety of the 50 subjects. The higher the score was, the stronger the anxiety was. After the experiment, the subjects filled in the SAS scale and the subjects filled in the questionnaire made by Qiang Cao, a member of the experimental group, were compared. Finally, it was found that the people with higher SAS score ( more serious 
anxiety ) showed the degree of anxiety and the possibility of self-transmission of anxiety when filling in the questionnaire made by the research group. And through the consent of volunteers under the premise of telephone interviews to understand the crowd around the subjects of their evaluation, and these evaluations are recorded and confidential to the subjects. The participants were asked to fill out the questionnaire. The results showed that $98 \%$ of the participants ( 49 people ) showed the same degree of influence of anxiety in the population as whether they would spread anxiety on various occasions and the evaluation of their surrounding people. It can be considered that the design of this questionnaire is reasonable and effective. Since anxiety is likely to spread in all kinds of people, the experimental group used the "questionnaire star" software to investigate 1200 people with different educational backgrounds, ages and identities. In order to ensure the randomness of the survey, the survey group randomly selected passers-by in multiple regions with different levels of wealth and education, and then interviewed some of the representative respondents. Representative respondents included those interested in the survey who were willing to accept the interview, those with different educational backgrounds, ages, and situations affected by anxiety, and those who were willing to accept the interview after the invitation of the research group. In order to find out the reasons why they were affected by anxiety in different occasions, and discuss them in order to find a solution to the problem of spreading anxiety in public occasions. After the end of the questionnaire survey, 1036 valid questionnaires were obtained after excluding filling out the short-term volume, with an effective rate of $86.3 \%$. The questionnaire was divided into 12 questions, which investigated the identity, educational background and the degree of anxiety in the crowd, including some questions to test whether the applicant lied during the filling process [ 33 ],For example, if the age of the respondents is obviously inconsistent with their education background, whether they have a job or not, or if they fill in contradictory questions, the 
questionnaire filled out by them will be regarded as invalid, [ 34 ] and the respondents will be excluded from the scope of this experiment. Through the preliminary questionnaire survey, it is finally found that the probability of the respondents producing and spreading anxiety at home ( $48.94 \%$ ) is significantly higher than that of the respondents producing and spreading anxiety in public places $(38.49 \%, \mathrm{p}<0.05)$. The probability of people affected by family anxiety $(54.93 \%)$ is higher than that of the respondents affected by stranger anxiety $(34.72 \%, p<0.05)$. A total of $69.83 \%$ of the respondents understand the concept of " anxiety spread in the population " and think they are vulnerable to the influence of anxiety spread in the population in the questionnaire. After the survey, the reliability analysis of the results of the survey is carried out by using SPSS software ( clonedbach index $=0.947$ ), and the results of this survey are reliable.

In order to ensure the accuracy of the survey and analyze the correlation between groups of data to obtain the correlation between the data, in order to analyze whether people in different environments will be affected by the atmosphere of anxiety in the environment, and the final results. The participants in this survey were randomly selected and willing to cooperate with the survey [ 35 ]. The identity of the subjects in this survey will be strictly confidential. Under this condition, the participants can be free from external interference. After the subjects completed the questionnaire, the survey group will select some representative respondents from the respondents for interviews, through interviews to understand whether they will be affected by anxiety in the crowd and whether they will spread anxiety in the crowd after being affected by anxiety or their own anxiety, and under what conditions they are more vulnerable to anxiety or spread anxiety . Finally, combined with the results of the questionnaire and interview content, the method to prevent the spread of anxiety in the crowd in various environments is found. After finding the reasons for the spread and generation of anxiety through analysis, the experimental group found a method to 
eliminate anxiety in the population and prevent the spread of anxiety in the population by making the anxiety-producing subjects observe the images of characters with positive emotions.

\section{Funding}

None

\section{Authors' contributions}

Y.Q. conceived the experiment(s), Q.C. and Q.Z. conducted the experiment(s), Q.C.and Y.Q. analysed the results. All authors reviewed the manuscript.

\section{Competing interests}

The authors declare that they have no competing interests.

\section{Availability of data and material}

Not applicable

\section{REFERENCES}

[1] Wilson, J. R., Scheutz, M. A model of esputing. And. Intelligent. Interaction. (ACII), Xi'an, China, 112-118, doi: 10.1109/ACII.2015.7344559 (2015)

[2] Wu, A. et al. Smartphone apps for depression and anxiety: a systematic review and meta-analysis of techniques to increase engagement. NPJ. Digit. Med.4, 20, doi:https://doi.org/10.1038/s41746-021-00386-8 (2021)

[3] Wang, Y. et al. Acute psychological effects of Coronavirus Disease 2019 outbreak among 
healthcare workers in China: a cross-sectional study. Transl. Psychiatry. 10, 348, https://doi.org/10.1038/s41398-020-01031-w (2020)

[4] Li, J. The status of the development of Eye-movement moving device and its application in psychology in China. 2010. International. Conference. on. Mechanic. Automation. and. Control. Engineering. Wuhan, China, 3153-3154, doi: 10.1109/MACE.2010.5535594 (2010)

[5] Schilling, M. et al. Do You Fake More Because of Your Neighbors? A Multi-level Study on Regional and Individual Predictors of Faking Intentions Across the USA. J. Bus.

Psychol. 36, 193-209, doi:https://doi-org.libezproxy.must.edu.mo/10.1007/s10869-019-09664-5(2021)

[6] Hager, R., Jones, C. (Eds.). Reproductive Skew in Vertebrates: Proximate and Ultimate Causes. Cambridge: Cambridge. University. Press. doi:10.1017/CBO9780511641954 (2009)

[7] Killeen, P.R. Predict, Control, and Replicate to Understand: How Statistics Can Foster the Fundamental Goals of Science. Perspect. Behav. Sci. 42, 109-132, https://doi-org.libezproxy.must.edu.mo/10.1007/s40614-018-0171-8 (2019)

[8] Evans, S. , Langberg, J. , Raggi, V. , Allen, J., Buvinger, E.C. Development of a school-based treatment program for middle school youth with ADHD. Journal. of. Attention. Disorders. 9, 343-353, doi: 10.1177/1087054705279305 (2005) 
[9] Kazdin, A.E. Evidence-based treatment and practice: New opportunities to bridge clinical research and practice, enhance the knowledge base, and improve patient care. American. Psychologist. 63, 146-159, doi: 10.1037/0003-066X.63.3.146 (2008)

[10]Cheng, Z., Wang, H., Shao, Z., Yang, Y. Bilateral Dynamic Negotiation Model Dominated by Personality Psychology and External Environment. 2010. International. Conference.on.

Computational. And. Information. Sciences. Chengdu, China, 351-354, doi: 10.1109/ICCIS.2010.92 (2010)

[11] Marzouki, Y., Aldossari, F.S. \& Veltri, G.A. Understanding the buffering effect of social media use on anxiety during the COVID-19 pandemic lockdown. Humanit. Soc. Sci. Commun. 8, 47, https://doi.org/10.1057/s41599-021-00724-x (2021)

[12] Vasser, M., Kängsepp, M., Kilvits, K., Kivisik, T., Aru, J. Virtual reality toolbox for experimental psychology — Research demo. 2015. IEEE. Virtual. Reality. (VR). Arles, France, 361-362, doi: 10.1109/VR.2015.7223445 (2015)

[13] Xiaomeng, H., Yue, C. Psychology and micro-blogging: Self-presentation, social interaction and social culture. 2010. IEEE. 2nd. Symposium. on. Web. Society. Beijing, China, 643-647, doi: 10.1109/SWS.2010.5607369 (2010)

[14] Starzak, T.B., Gray, R.D. Towards ending the animal cognition war: a three-dimensional model 
of causal cognition. Biol. Philos. 36, 9,

https://doi-org.libezproxy.must.edu.mo/10.1007/s10539-021-09779-1 (2021)

[15] Bonawitz, E. et al. Just do it? Toddlers' ability to integrate prediction, action and expectation about contact relations. Cognition. 115,104-117, doi: 10.1016/j.cognition.2009.12.001 (2010)

[16] Mihailov, A., Philippe, C., Gloaguen, A. et al. Cortical signatures in behaviorally clustered autistic traits subgroups: a population-based study. Transl Psychiatry 10, 207 (2020). https://doi.org/10.1038/s41398-020-00894-3

[17] Luo, X. The Application of Management Experiment on Humanization Management in Real Experience Practice and Field Work of Students. 2010. 3rd. International. Conference. on. Information. Management., Innovation. Management. and. Industrial. Engineering. Kunming, China, 483-485, doi: 10.1109/ICIII.2010.122 (2010)

[18] Hortensius, R., Hekele, F., Cross, E.S. The Perception of Emotion in Artificial Agents. In. IEEE. Transactions. on. Cognitive. and. Developmental. Systems. vol. 10, no. 4, 852-864, doi: 10.1109/TCDS.2018.2826921 (2018)

[19] Weinbrecht, A., Niedeggen, M., Roepke, S. Renneberg, B. Processing of increased frequency of social interaction in social anxiety disorder and borderline personality disorder. Sci.

Rep. 11, 5489, https://doi.org/10.1038/s41598-021-85027-6 (2021)

[20] Amin, S.U., Hossain M.S. Edge Intelligence and Internet of Things in Healthcare: A Survey. 
In. IEEE. Access. 9, 45-59, doi: 10.1109/ACCESS.2020.3045115 (2021)

[21] Roy, A., Nikolitch, K., McGinn, R. et al. A machine learning approach predicts future risk to suicidal ideation from social media data. npj Digit. Med. 3, 78 (2020).

https://doi.org/10.1038/s41746-020-0287-6

[22] Beck, A. How an anomalous finding led to a new system of psychotherapy. Nat Med 12, 1139-1141 (2006). https://doi.org/10.1038/nm1006-1139

[23] Yeh, S.C., Li, Y.Y., Zhou,C., Chiu,P. H., Chen,J.W. Effects of Virtual Reality and Augmented Reality on Induced Anxiety. In. IEEE. Transactions. On. Neural. Systems. and. Rehabilitation. Engineering.26, 1345-1352, doi: 10.1109/TNSRE.2018.2844083 (2018)

[24] Wu, A., et al. Smartphone apps for depression and anxiety: a systematic review and meta-analysis of techniques to increase engagement. Npj. Digit. Med. 4, 20, https://doi.org/10.1038/s41746-021-00386-8 (2021)

[25] BLACKMAN, D. Effects of Drugs on Conditioned “Anxiety”. Nature. 217, 769-770, https://doi.org/10.1038/217769a0 (1968)

[26] Zhang, G.W., et al. Medial preoptic area antagonistically mediates stress-induced anxiety and parental behavior. Nat .Neurosci . 24, 516-528, https://doi.org/10.1038/s41593-020-00784-3 (2021) 
[27] Velurajah, R., Brunckhorst, O., Waqar, M., McMullen, I., Ahmed, K. Erectile dysfunction in patients with anxiety disorders: a systematic review. Int. J. Impot. Res.

https://doi.org/10.1038/s41443-020-00405-4 (2021)

[28] Gerontology: Public Anxiety about

Ageing. Nature. 226, 895-896, https://doi.org/10.1038/226895a0 (1970)

[29] Li,Y., Huang, X., Zhao, G. Joint Local and Global Information Learning With Single Apex

Frame Detection for Micro-Expression Recognition. In. IEEE .Transactions. On. Image .

Processing. 30, 249-263, doi: 10.1109/TIP.2020.3035042 (2021)

[30] Li, W., et al. The prevalence of depressive and anxiety symptoms and their associations with quality of life among clinically stable older patients with psychiatric disorders during the COVID-19 pandemic. Transl. Psychiatry. 11, 75, https://doi.org/10.1038/s41398-021-01196-y (2021)

[31] Lomas, T. The dimensions of prosociality: a cross-cultural lexical analysis. Curr. Psychol. 40,1336-1347, https://doi-org.libezproxy.must.edu.mo/10.1007/s12144-018-0067-5 (2021)

[32] Mehrotra, S. Synergy between Positive Psychology and Indian Psychology? Reflections on Barriers and Pathways. Psychol. Stud. 59, 113-115, https://doi-org.libezproxy.must.edu.mo/10.1007/s12646-014-0257-7 (2014) 
[33] Christopher, J.C. Putting "Positive" and "Psychology" In Perspective: The Role of Indian Psychology. Psychol. Stud. 59, 110-112,

https://doi-org.libezproxy.must.edu.mo/10.1007/s12646-014-0256-8 (2014)

[34] Bellow, G. Self psychology and ego psychology: A historical perspective. Clin. Soc. Work.

J. 14, 199-212, https://doi-org.libezproxy.must.edu.mo/10.1007/BF00755853 (1986)

[35] Renn, B.N., Hoeft, T.J., Lee, H.S., Bauer, A.M. Areán PA. Preference for in-person psychotherapy versus digital psychotherapy options for depression: survey of adults in the U.S. npj.

Digital. Med. 2, 6 , https://doi.org/10.1038/s41746-019-0077-1 (2019) 